${ }^{+} \stackrel{+}{+}++^{+}$La revue pour l'histoire du CNRS

POUR LHISTORE DU CNRS $\quad 24 \mid 2009$

Soixante-dixième anniversaire du CNRS

\title{
Un fléau nommé paludisme
}

Bernard Meunier

\section{OpenEdition}

Journals

Édition électronique

URL : https://journals.openedition.org/histoire-cnrs/9105

DOI : 10.4000/histoire-cnrs.9105

ISSN : 1955-2408

Éditeur

CNRS Éditions

Édition imprimée

Date de publication : 5 octobre 2009

ISSN : 1298-9800

Référence électronique

Bernard Meunier, « Un fléau nommé paludisme », La revue pour l'histoire du CNRS [En ligne], 24 | 2009, mis en ligne le 05 octobre 2009, consulté le 20 mai 2021. URL : http://journals.openedition.org/ histoire-cnrs/9105; DOI : https://doi.org/10.4000/histoire-cnrs.9105

Ce document a été généré automatiquement le 20 mai 2021.

Comité pour l'histoire du CNRS 


\title{
Un fléau nommé paludisme
}

\author{
Bernard Meunier
}

1 La vie d'un chercheur est longue : la passion pour la recherche n'a pas de raison de s'exprimer de manière strictement linéaire. Beaucoup d'entre nous suivent des chemins qui peuvent être considérés comme s'éloignant d'une recherche fondamentale orthodoxe, en fait définie comme telle par les collègues qui se considèrent comme étant «au coeur e la discipline». Je dois avouer que je n'ai jamais résisté au plaisir de regarder à côté de la grande route, dès lors que cela me permettait d'élargir les horizons et d'explorer le continuum entre la recherche fondamentale et ses applications. La recherche et l'innovation sont comme les deux faces d'une même pièce de monnaie, elles sont inséparables et doivent être aussi belles l'une que l'autre. A priori, rien dans mon parcours de chercheur entre 1969 et 1995 ne laissait à penser que la recherche de nouveaux antipaludiques allait tenir une part aussi importante dans mon travail à partir de 1995- 1996. De 1969 à 1972, à l'université de Montpellier, j'ai travaillé sur l'activation catalytique de réactifs de Grignard à l'aide de complexes du nickel et du titane dans le laboratoire de Robert Corriu, dédié à l'époque à la chimie organique du silicium.

2 En janvier 1973, mon recrutement au CNRS dans l'équipe de Hugh Felkin, complète cette "période chimie organométallique» de ma carrière. C'est aussi celle de la préparation et la caractérisation par diffraction des rayons $\mathrm{X}$ de la première molécule à liaison covalente entre un atome de fer et un atome de magnésium, un réactif de Grignard inorganique. Au-delà du plaisir de cette recherche fondamentale ayant peu de liens avec la réalité de l'innovation, j'ai eu la chance de travailler à l'Institut de chimie des substances naturelles du CNRS, situé au cœur du campus très agréable de Gif-surYvette en vallée de Chevreuse.

3 Évoluer dans un institut du CNRS connu pour ses recherches en chimie biologique et thérapeutique a largement influencé mon attrait pour ce domaine. Cette époque était celle des recherches de Pierre Potier et de son équipe sur des molécules qui deviendront des médicaments anticancéreux, la navelbine et le taxotère, développés plus tard par les groupes pharmaceutiques Pierre Fabre et Rhône-Poulenc. De cette période giffoise, j'ai gardé la joie de la rencontre entre la chimie des ions métalliques et 
la biochimie des métalloenzymes. Ayant manipulé de l'eau de Javel ( $\mathrm{NaOCl}$ ) pour éliminer des phosphines très odorantes, c'est très naturellement que je me suis tourné vers ce réactif pour créer des espèces métal-oxo capables de mimer les entités actives des cytochromes P450 ou des peroxydases. Des premières idées sur les «donneurs d'atomes d'oxygène " à Gif en 1977, à la réalisation à Toulouse en 1980, une période d'induction a été occupée à la pratique de la cristallographie dans l'équipe de Claudine Pascard à Gif, puis dans celle de Keith Prout à l'université d'Oxford (1977-1978). Ce changement de thème de recherche, orienté sur le rôle des ions métalliques dans les transferts catalytiques d'atomes d'oxygène ou d'électrons dans les systèmes chimiques ou biochimiques, a été l'occasion d'une mobilité géographique et de la création d'une petite équipe de recherche, au sein du Laboratoire de chimie de coordination à Toulouse, situé sur le même campus que le Laboratoire de pharmacologie et toxicologie fondamentales. Ces deux autres laboratoires étaient des unités propres du CNRS, comme l'ICSN.

4 En 1995, l'équipe était en plein développement, forte d'une quinzaine de collègues, jeunes chercheurs et étudiants, avec une réputation avérée dans le domaine des oxydations biomimétiques et de l'activation enzymatique de médicaments, lorsque le hasard de la bibliographie m'amena à lire dans le journal de la société chimique américaine (JACS), un article d'un collègue américain, dont le nom évoquait pour moi des travaux en chimie organométallique dans les années 1980. Ayant démarré mon activité de chercheur avant l'ère de l'informatique et d'Internet, j'avais gardé (de moins en moins maintenant!) cette pratique de passer à la bibliothèque et de lire une quinzaine de périodiques en chimie ou en biologie considérés comme étant les plus réputés dans mes domaines de recherche. Cet article de Gary Posner, pour le nommer, indiquait qu'une molécule antipaludique, l'artémisinine, était capable de créer une espèce métal-oxo avec des complexes métalliques contenant du fer. Bigre!

Connaissant la difficulté à créer des espèces métal-oxo avec des peroxydes organiques dont la liaison oxygène/oxygène est trop peu dissymétrique pour être rompue de manière hétérolytique (condition nécessaire pour produire une entité métal-oxo de haut degré à partir d'un peroxyde), j'ai regardé avec intérêt la structure chimique de l'artémisinine, produit naturel extrait de l'armoise Artemisia annua et utilisé comme antipaludique dans la médecine traditionnelle chinoise. Voilà comment je suis passé de la chimie des espèces métal-oxo à des recherches sur le paludisme. Anne Robert, chargée de recherche dans mon équipe, accepta alors avec enthousiasme de regarder l'interaction de ce médicament ayant un pont endoperoxyde avec des métalloporphyrines de manganèse et de fer. Dès 1997, nous montrons que cet antipaludique est facilement activé par transfert mono-électronique à partir d'un site métallique réduit et conduit à un radical carboné capable d'alkyler des modèles d'hème ou même l'hème lui-même. Cette étude du mécanisme d'action d'un médicament antipaludique va s'amplifier pour aller jusqu'à la conception de nouvelles molécules hybrides à activité duale, «les trioxaquines ». Une de ces molécules, la PA1103, est maintenant en développement industriel grâce à l'action concertée de Palumed (jeune société créé ${ }^{1}$ spécialement en 2000 pour développer ces antipaludiques) et de SanofiAventis (B. Meunier, Acc. Chem. Res., 2008, vol. 41, pp. 69-79). C'est ainsi que l'on passe insensiblement, en un peu plus de 30 ans, de la chimie du silicium à celle des antipaludiques. Je ne regrette pas cette méthode de glissement d'un sujet de recherche à un autre, elle m'a apporté beaucoup de satisfactions intellectuelles et la joie de voir le passage d'une recherche fondamentale à la très longue route de l'innovation dans le 
domaine des médicaments antipaludiques. Il va falloir attendre encore quelques années pour savoir si l'une de ces trioxaquines pourra devenir ou non un nouvel outil de l'arsenal thérapeutique de la lutte contre le paludisme. Heureusement, la durée de vie s'allonge, nous pouvons donc mener des projets de longue haleine !

6 Quassia, Kwasi (Quassia amara L.). Jeune plant et jeunes feuilles (les jeunes feuilles sont rouges) d'un petit arbuste de la forêt amazonienne, plus connu sous le nom de quinquina de Cayenne. Des chercheurs viennent d'isoler un des principes actifs antipaludiques de la tisane de "Quassia », un remède utilisé traditionnellement par les populations locales de Guyane française. (C) CNRS Photothèque/Didier Stien

\section{NOTES}

1. Dans le cadre de la loi de 1999 sur la recherche et l'innovation.

\section{RÉSUMÉS}

De la recherche fondamentale à l'innovation thérapeutique, des molécules biomimétiques aux antipaludiques... Rien, en apparence, ne prédestinait Bernard Meunier à une telle trajectoire. Pourtant, avec Palumed SA, la société de chimie thérapeutique qu'il a créée en 2000, il a fait preuve d'une polyvalence inestimable et accompagné un transfert technologique réussi.

\section{AUTEUR}

\section{BERNARD MEUNIER}

Bernard Meunier, membre de l'Académie des sciences et ancien président du CNRS, a co-dirigé le Laboratoire de chimie de coordination. Il a créé la société Palumed. 